\title{
MAXIMA OF MOVING SUMS IN A POISSON RANDOM FIELD
}

\author{
HOCK PENG CHAN, * National University of Singapore
}

\begin{abstract}
In this paper we examine the extremal tail probabilities of moving sums in a marked Poisson random field. These sums are computed by adding up the weighted occurrences of events lying within a scanning set of fixed shape and size. We also provide an alternative representation of the constants of the asymptotic formulae in terms of the occupation measure of the conditional local random field at zero, and extend these representations to the constants of asymptotic tail probabilities of Gaussian random fields.
\end{abstract}

Keywords: Change of measure; Gaussian process; large deviations; marked Poisson process; moving sums; random field; scan statistics

2000 Mathematics Subject Classification: Primary 60F10

Secondary 60G10; 60G55

\section{Introduction}

The maxima of moving averages in Gaussian random fields in dimension $d>1$ was studied in [19] and [21], with applications in imaging and signal detection. Two key techniques used are (i) the Karhunen-Loève expansion with the volume of tube formula and (ii) the Euler characteristic; see [1] for an overview, and [24] and [25] for more recent developments.

The maxima of moving sums in Poisson random fields, more commonly known as scan statistics in the statistical literature, is also used widely in molecular biology, epidemiology, geostatistics, and image analysis (cf. [4], [8], [9], and [11]), but the tail probability approximations are in comparison not as well developed for $d>1$. While the tail probabilities of these sums have been studied in [3], [14], and [15], restrictions to rectangular scanning sets have been imposed for analytical convenience.

We set out here to study the tail probabilities of the maxima of moving sums for a wider choice of scanning sets. A theory parallel to the study of tail probabilities in Gaussian or Gaussianlike random fields in the classical framework of Pickands [16] and Qualls and Watanabe [18] (see also [7] and [17]) is first developed. Motivated by recent developments in molecular biology (see, for example, [8]), we consider a more general marked Poisson random field. This generalization entails careful consideration of overshoots in special cases of scanning sets that is not required in Poisson random fields. Albin [2] and Berman [5] studied tail probabilities of stationary processes, but their limiting results are of a different type and do not apply here. Kabluchko and Spodarev [13] considered scan statistics of Lévy noises and marked empirical processes, with the scan over all cubic subsets of $[0,1]^{d}$.

The main results of this paper are Theorems 2.1 and 2.2. We illustrate their applications with examples in Section 3. Theorem 2.1 provides an expression of the asymptotic tail probability and the statement of the result requires a description of an induced local random field around

Received 18 September 2008; revision received 20 March 2009.

* Postal address: Department of Statistics and Applied Probability, Faculty of Science, National University of Singapore, Science Drive 1, 119260, Singapore. Email address: stachp@nus.edu.sg 
the boundary of the scanning set. The proof of Theorem 2.1 is given in Section 4. Lemma 4.1 is the basic building block of Theorem 2.1, providing the extremal tail probability over a local domain by using a change of measure approach. We then show how these building blocks can be combined together to provide the tail probability of the maxima of the sums over the whole domain, via an adaption of the Pickands-Qualls-Watanabe technique. Theorem 2.2 provides an alternative representation of the asymptotic constants in terms of the occupation measure of the local random field at zero. The proof is given in Section 5. We extend the representation to Gaussian random fields in Section 6. These representations provide simple upper and lower bounds of the asymptotic constants; see the examples in Sections 3 and 6. We consolidate the technical details in the appendices.

\section{Main results}

For vectors $t=\left(t_{1}, \ldots, t_{d}\right)$ and $u=\left(u_{1}, \ldots, u_{d}\right)$, and real number $b$, we shall let $t \geq u$ denote $t_{j} \geq u_{j}$ for all $j$ and let $t \geq b$ denote $t_{j} \geq b$ for all $j$. Let $\sigma_{k}(\cdot), k=d$ or $k=$ $d-1$, denote the Lebesgue or Hausdorff measure of a $k$-dimensional manifold in $\mathbb{R}^{d}$. For any $A \subset \mathbb{R}^{d}$, real number $b$, and $t \in \mathbb{R}^{d}$, let $\# A$ denote the number of elements in $A$ and $t+b A=\{t+b a: a \in A\}$. Also, let $\|t\|=\left(t_{1}^{2}+\cdots+t_{d}^{2}\right)^{1 / 2}$ and $\|t\|_{\infty}=\max _{1 \leq j \leq d}\left|t_{j}\right|$.

Let $B$ be a bounded subset of $\mathbb{R}^{d}$ with boundary $\partial B$ that can be expressed as a finite union of smooth $(d-1)$-dimensional submanifolds possibly with boundaries (see [22, p. 113] for the definition), satisfying $\sigma_{d-1}(\partial B)<\infty$. For example, if $B=\left\{t:\|t\|_{\infty} \leq 1\right\}$, a cube of length 2 centered at 0 , then $\partial B$ is a union of $2 d$ faces, each a smooth $(d-1)$-dimensional submanifold with boundary.

Let $\left\{t_{i}: i \geq 1\right\}$ be a homogeneous Poisson point process on $\mathbb{R}^{d}$ with intensity $\lambda>0$, and let $X_{1}, X_{2}, \ldots$ be independent and identically distributed (i.i.d.) random variables having distribution $F$ and independent of the Poisson point process. Let $\mathcal{X}=\left\{\left(t_{i}, X_{i}\right): i \geq 1\right\}$ denote the marked point process. Let $\mu=\mathrm{E}\left(X_{1}\right)$ and $M(\theta)=\mathrm{E}\left(\mathrm{e}^{\theta X_{1}}\right)$. Assume that $\Theta=\{\theta: M(\theta)<$ $\infty\}$ is an open neighborhood of 0 . For any $A \subset \mathbb{R}^{d}$, define the sum $S(A)=\sum_{t_{i} \in A} X_{i}$. Let $D$ be a Jordan measurable (bounded) subset of $\mathbb{R}^{d}$, and let $c>\mu \sigma_{d}(B)$. We analyze here the tail probability

$$
p_{\lambda}=\mathrm{P}_{\lambda}\left(\sup _{v \in D} S(v+B) \geq \lambda c\right) \quad \text { as } \lambda \rightarrow \infty .
$$

Through an appropriate transformation, we can also look at the limiting probability of $p_{\lambda}$ as one involving fixed Poisson rate $\lambda_{0}>0$ and increasingly large scanning sets. Let $g_{\lambda}=\left(\lambda / \lambda_{0}\right)^{1 / d}$ be the scaling constants. Then $p_{\lambda}=\mathrm{P}_{\lambda_{0}}\left(\sup _{v \in g_{\lambda} D} S\left(v+g_{\lambda} B\right) \geq \lambda c\right)$. For notational simplicity, the analysis here looks at $p_{\lambda}$ in terms of (2.1), but in terms of practical use, the asymptotics in terms of increasing scanning sets is sometimes more appropriate.

We will now proceed with the description of a limiting local random field $Y$ that is derived from both the distribution $F$ and the geometry of the boundary $\partial B$. Let $\theta_{c}>0$ and distribution $F_{c}$ satisfy

$$
M^{\prime}\left(\theta_{c}\right)=\frac{c}{\sigma_{d}(B)} \quad \text { and } \quad F_{c}(\mathrm{~d} x)=\frac{\mathrm{e}^{\theta_{c} x} F(\mathrm{~d} x)}{M\left(\theta_{c}\right)},
$$

where a prime here denotes the first derivative. By (2.2), a random variable having distribution $F_{c}$ has mean $M^{\prime}\left(\theta_{c}\right) / M\left(\theta_{c}\right)=c /\left[\sigma_{d}(B) M\left(\theta_{c}\right)\right]$ and, hence, a marked Poisson process with intensity $\lambda M\left(\theta_{c}\right)$ and mark distribution $F_{c}$ inside $B$ will satisfy $\mathrm{E}_{*}[S(B)]=\lambda c$.

Consider the product space $\partial B \times \mathbb{R}$ with measure induced from the $(d-1)$-dimensional Hausdorff measure on $\partial B$ and Lebesgue measure on $\mathbb{R}$. Let $\left\{v_{i}: i \geq 1\right\}$ be a nonhomogeneous 
Poisson point process on $\partial B \times \mathbb{R}$ with intensity 1 on $\partial B \times[0, \infty)$ and intensity $M\left(\theta_{c}\right)$ on $\partial B \times(-\infty, 0)$. Let $Z_{1}, Z_{2}, \ldots$ be independent random variables (marks) conditioned on this nonhomogeneous point process, with $Z_{i} \sim F$ if $v_{i} \in \partial B \times[0, \infty)$ and $Z_{i} \sim F_{c}$ if $v_{i} \in \partial B \times(-\infty, 0)$. Let $\mathrm{Q}_{\lambda}$ be a probability measure under which $\mathcal{X}=\left\{\left(t_{i}, X_{i}\right): i \geq 1\right\}$ is a nonhomogenous marked point process with intensity $\lambda M\left(\theta_{c}\right)$ and mark distribution $F_{c}$ inside $B$, and intensity $\lambda$ and mark distribution $F$ outside $B$. The measure $\mathrm{Q}_{\lambda}$ describes the local behavior of $\mathcal{X}$ near $\partial B$ under the original probability measure $\mathrm{P}_{\lambda}$ in which $\mathcal{X}$ is homogeneous, when conditioned on $\{S(B) \geq \lambda c\}$. Let $\partial B^{(\delta)}=\left\{u \in \mathbb{R}^{d}:\|u-t\| \leq \delta\right.$ for some $\left.t \in B\right\}$. For any $m>0$ and large $\lambda$, we can apply a $\lambda$-scaling transformation such that $\mathcal{X}$ under $\mathrm{Q}_{\lambda}$ and restricted to $\partial B^{\left(m \lambda^{-1}\right)}$ tends to $\mathcal{Z}$ restricted to $\partial B \times[-m, m]$ as $\lambda \rightarrow \infty$.

Let $n_{t}$ be the unit normal vector of $t \in \partial B$ pointing away from $B$, and let a dot denote the scalar or inner product of two vectors. Let

$$
Y^{(j)}(u)=\sum_{\left\{i: v_{i}^{(j)} \in A_{u}^{(j)}\right\}} Z_{i} \quad \text { for } j=1,2,
$$

where

$$
A_{u}^{(1)}=\bigcup_{\left\{t \in \partial B: n_{t} \cdot u>0\right\}} t \times\left[0, n_{t} \cdot u\right) \quad \text { and } \quad A_{u}^{(2)}=\bigcup_{\left\{t \in \partial B: n_{t} \cdot u<0\right\}} t \times\left[n_{t} \cdot u, 0\right) .
$$

We define the limiting local random field by

$$
Y(u)=Y^{(1)}(u)-Y^{(2)}(u), \quad u \in \mathbb{R}^{d} .
$$

Let

$$
I\left(=I_{c}\right)=\theta_{c} c-\sigma_{d}(B)\left[M\left(\theta_{c}\right)-1\right] .
$$

It follows from Theorem 2.1, below, that $I=-\lim _{\lambda \rightarrow \infty} \lambda^{-1} \log p_{\lambda}$ and, hence, it is the large deviation rate of the tail probability $p_{\lambda}$. If there exists $\eta>0$ such that $F$ is concentrated on $\pm \eta, \pm 2 \eta, \ldots$, then we say that $F$ is arithmetic. The largest $\eta$ with this property is called the span of $F$; cf. Section 5.2 of [10]. If such $\eta$ does not exist then we say that $F$ is nonarithmetic. Let $\lfloor\cdot\rfloor$ denote the greatest integer function, and let a double prime denote the second derivative of a function.

Theorem 2.1. Define $x_{\lambda}=\theta_{c}(\lambda c-\eta\lfloor\lambda c / \eta\rfloor)$ if $F$ is arithmetic with span $\eta$ and $x_{\lambda}=0$ if $F$ is nonarithmetic. Let

$$
K_{m}= \begin{cases}\eta\left[\left(1-\mathrm{e}^{-\eta \theta_{c}}\right)^{-1}+\sum_{\ell \in \eta \mathbb{Z}^{+}} \mathrm{e}^{\theta_{c} \ell} \mathrm{P}\left(\sup _{0 \leq u \leq m} Y(u) \geq \ell\right)\right] & \text { if F arithmetic with span } \eta, \\ \theta_{c}^{-1}+\int_{0}^{\infty} \mathrm{e}^{\theta_{c} y} \mathrm{P}\left(\sup _{0 \leq u \leq m} Y(u) \geq y\right) \mathrm{d} y & \text { if F nonarithmetic. }\end{cases}
$$

Then

$$
K=\lim _{m \rightarrow \infty} m^{-d} K_{m} \text { is a well-defined positive and finite constant } .
$$

Moreover, as $\lambda \rightarrow \infty$,

$$
\begin{aligned}
p_{\lambda} & =\mathrm{P}_{\lambda}\left(\sup _{v \in D} S(v+B) \geq \lambda c\right) \\
& \sim\left[2 \pi \sigma_{d}(B) M^{\prime \prime}\left(\theta_{c}\right)\right]^{-1 / 2} \mathrm{e}^{-\lambda I+x_{\lambda}} \lambda^{d-1 / 2} \sigma_{d}(D) K .
\end{aligned}
$$


Remark 2.1. By Jordan measurability of $D$, as $a \rightarrow 0$,

$$
\#\left\{k \in(a \mathbb{Z})^{d}: \prod_{j=1}^{d}\left[k_{j}, k_{j}+a\right) \subset D\right\} \sim \#\left\{k \in(a \mathbb{Z})^{d}: \prod_{j=1}^{d}\left[k_{j}, k_{j}+a\right) \cap D \neq \varnothing\right\} .
$$

Relation (2.8) still holds if $D$ is replaced by domains $D_{\lambda}$ that depend on $\lambda$, provided that (2.9) holds with $D$ replaced by $D_{\lambda}$ and $a$ replaced by $m \lambda^{-1}$, with limit $\lambda \rightarrow \infty$ for all large $m$, and

$$
\lim _{\lambda \rightarrow \infty} \lambda^{-1} \log \left[\sigma_{d}\left(D_{\lambda}\right)\right]=0
$$

However, even without condition (2.10), the relation

$$
\begin{aligned}
\mathrm{P}_{\lambda}\left(\sup _{v \in D_{\lambda}} S(v+B) \geq \lambda c\right) \\
\quad \sim 1-\exp \left\{-\left[2 \pi \sigma_{d}(B) M^{\prime \prime}\left(\theta_{c}\right)\right]^{-1 / 2} \mathrm{e}^{-\lambda I+x_{\lambda}} \lambda^{d-1 / 2} \sigma_{d}\left(D_{\lambda}\right) K\right\}
\end{aligned}
$$

still holds.

We now provide an alternative expression of $K$ which follows from a different proof of (2.8). This representation is less intuitive when compared to (2.6) and (2.7), but it has the advantage of being able to provide simple bounds for $K$. Let $\sigma_{d}^{-1}(\cdot)$ denote the reciprocal of $\sigma_{d}(\cdot)$.

Theorem 2.2. The constant

$$
K=\chi_{c}^{-1} \mathrm{E}\left(\left[\left(1-\exp \left\{\theta_{c} \sup \{Y(u): Y(u)<0\}\right\}\right] \sigma_{d}^{-1}(\{u: Y(u)=0\})\right),\right.
$$

where $\chi_{c}=\eta^{-1}\left(1-\mathrm{e}^{-\eta \theta_{c}}\right)$ when $F$ is arithmetic with span $\eta$ and $\chi_{c}=\theta_{c}$ when $F$ is nonarithmetic.

\section{Examples}

We first discuss two interesting cases of Theorem 2.1. In Example 3.1 we consider $F$ to be degenerate at 1. Then (2.8) simplifies to (3.2), below, an extension of tail probability relations in Poisson random fields obtained earlier by other authors to nonrectangular scanning sets. In Example 3.2 we consider rectangular scanning sets on a marked Poisson random field. We show here that an overshoot constant derived from $F$ plays an important role in the tail approximations. When $F$ is degenerate at 1 , that is, for Poisson random fields rather than marked Poisson random fields, the overshoot constant is equal to 1 and disappears from the resulting formula. We then show in Examples 3.3 and 3.4 how the alternative expression in Theorem 2.2 leads to simple upper and lower bounds of $K$.

Example 3.1. Let $F$ be concentrated at 1 . Then $S(v+B)$ counts the number of points $t_{i}$ lying inside $v+B$. Since $M(\theta)=M^{\prime}(\theta)=M^{\prime \prime}(\theta)=\mathrm{e}^{\theta}$, it follows from (2.2) and (2.5) that

$$
\mathrm{e}^{I}=\left(\frac{c}{\sigma_{d}(B)}\right)^{c} \mathrm{e}^{-c+\sigma_{d}(B)}
$$

Substituting (2.2) and (3.1) into (2.8) and noting that $F$ is arithmetic with span 1, we obtain

$$
p_{\lambda} \sim(2 \pi)^{-1 / 2} \sigma_{d}^{\lfloor\lambda c\rfloor}(B) c^{-\lfloor\lambda c\rfloor-1 / 2} \mathrm{e}^{\lambda c-\lambda \sigma_{d}(B)} \lambda^{d-1 / 2} \sigma_{d}(D) K \quad \text { as } \lambda \rightarrow \infty .
$$


Example 3.2. Let $B=\prod_{k=1}^{d}\left[0, b_{k}\right]$ with $b_{k}>0$ for all $k$. Since $\partial B$ is a union of $2 d$ faces, with a pair of them orthogonal to each coordinate vector, by (2.3) and (2.4),

$$
Y(u)=\sum_{k=1}^{d}\left[Y_{k}^{(1)}\left(u_{k}\right)-Y_{k}^{(2)}\left(u_{k}\right)\right],
$$

where $Y_{1}^{(1)}, \ldots, Y_{d}^{(1)}, Y_{1}^{(2)}, \ldots, Y_{d}^{(2)}$ are independent one-dimensional compound Poisson processes. The process $Y_{k}^{(1)}, 1 \leq k \leq d$, is constructed from a marked point process with intensity $\prod_{\ell \neq k} b_{\ell}$ (the surface area of the face of $B$ orthogonal to the $k$ th coordinate vector) and mark distribution $F$. The process $Y_{k}^{(2)}, 1 \leq k \leq d$, is constructed from a marked point process with intensity $M\left(\theta_{c}\right) \prod_{\ell \neq k} b_{\ell}$ and mark distribution $F_{c}$. If $X$ is a random variable with distribution $F_{c}$, we shall let $\bar{F}_{c}$ denote the distribution of $-X$. We define $\bar{F}$ in a similar manner. First consider $F$ nonarithmetic, and let $Y_{k}=Y_{k}^{(1)}-Y_{k}^{(2)}$. Then, by (2.6) and as $\mathrm{P}\left(\sup _{0 \leq u \leq m} \sum_{k=1}^{d} Y_{k}\left(u_{k}\right) \geq y\right)=1$ for $y \leq 0$,

$$
\begin{aligned}
K_{m} & =\int_{-\infty}^{\infty} \mathrm{e}^{\theta_{c} y} \mathrm{P}\left(\sup _{0 \leq u \leq m} \sum_{k=1}^{d} Y_{k}\left(u_{k}\right) \geq y\right) \mathrm{d} y \\
& =\theta_{c}^{-1} \mathrm{E}\left(\exp \left(\theta_{c} \sum_{k=1}^{d} \sup _{0 \leq u_{k} \leq m} Y_{k}\left(u_{k}\right)\right)\right) \\
& =\theta_{c}^{d-1} \prod_{k=1}^{d} \int_{-\infty}^{\infty} \mathrm{e}^{\theta_{c} y} \mathrm{P}\left(\sup _{0 \leq u_{k} \leq m} Y_{k}\left(u_{k}\right) \geq y\right) \mathrm{d} y .
\end{aligned}
$$

Since $Y_{k}^{(1)}$ and $Y_{k}^{(2)}$ are independent compound Poisson processes, it follows that $Y_{k}\left(u_{k}\right)=$ $\sum_{j=1}^{N_{k}\left(u_{k}\right)} U_{k j}$, where $N_{k}$ is a Poisson process with rate parameter $\left(\prod_{\ell \neq k} b_{\ell}\right)\left[1+M\left(\theta_{c}\right)\right]$ and $U_{k 1}, U_{k 2}, \ldots$ are i.i.d. random variables independent of $N_{k}$ such that

$$
\mathrm{P}\left(U_{k 1} \in \mathrm{d} u\right)=\frac{M\left(\theta_{c}\right) \bar{F}_{c}(\mathrm{~d} u)+F(\mathrm{~d} u)}{1+M\left(\theta_{c}\right)} .
$$

Let $\mathrm{P}_{*}$ be a probability measure under which the distribution of $N_{k}$ is unchanged, and let $U_{k 1}$, $U_{k 2}, \ldots$ be i.i.d. random variables independent of $N_{k}$ satisfying

$$
\mathrm{P}_{*}\left(U_{k 1} \in \mathrm{d} u\right)=\frac{\bar{F}(\mathrm{~d} u)+M\left(\theta_{c}\right) F_{c}(\mathrm{~d} u)}{1+M\left(\theta_{c}\right)} .
$$

By (2.2), (3.4), and (3.5),

$$
\frac{\mathrm{dP}}{\mathrm{dP}} U_{k 1}=\exp \left\{\theta_{c} U_{k 1}\right\}
$$

Suppressing the notation $k$, let $R_{\ell}=U_{1}+\cdots+U_{\ell}$ and $\tau_{y}=\inf \left\{\ell \geq 1: R_{\ell} \geq y\right\}$. Define the overshoot constant

$$
v_{c}=\lim _{y \rightarrow \infty} \mathrm{E}_{*}\left(\exp \left\{-\theta_{c}\left(R_{\tau_{y}}-y\right)\right\}\right),
$$

where $E_{*}$ denotes expectation with respect to $P_{*}$. See [23], [26]-[28], and Chapter 8 of [20] for analytical expressions of $v_{c}$ and also of related constants for sample size calculations. 
By (3.5)-(3.7),

$$
\begin{aligned}
\int_{-\infty}^{\infty} & \mathrm{e}^{\theta_{c} y} \mathrm{P}\left(\sup _{0 \leq u_{k} \leq m} Y_{k}\left(u_{k}\right) \geq y\right) \mathrm{d} y \\
& =\theta_{c}^{-1}+\int_{0}^{\infty} \mathrm{E}_{*}\left(\exp \left\{\theta_{c}\left(y-R_{\tau_{y}}\right)\right\} \mathbf{1}_{\left\{\sup _{0 \leq u_{k} \leq m} Y_{k}\left(u_{k}\right) \geq y\right\}}\right) \mathrm{d} y \\
& \sim v_{c} \mathrm{E}_{*}\left(\sup _{0 \leq u_{k} \leq m} Y_{k}\left(u_{k}\right)\right) \\
& \sim v_{c} m\left[c \sigma_{d}^{-1}(B)-\mu\right] \prod_{\ell \neq k} b_{\ell},
\end{aligned}
$$

noting that, by (2.2), under $F_{c}, \mathrm{E}_{c}\left(X_{1}\right)=M^{\prime}\left(\theta_{c}\right) / M\left(\theta_{c}\right)=c /\left[\sigma_{d}(B) M\left(\theta_{c}\right)\right]$, and by definition, under $F, \mathrm{E}\left(X_{1}\right)=\mu$. Substitute (3.8) into (3.3). Then, by (2.7) and (2.8),

$$
\begin{aligned}
p_{\lambda} \sim & {\left[2 \pi \sigma_{d}(B) M^{\prime \prime}\left(\theta_{c}\right)\right]^{-1 / 2} \mathrm{e}^{-\lambda I+x_{\lambda}} \lambda^{d-1 / 2} \sigma_{d}(D)\left\{v_{c}\left[c \sigma_{d}^{-1}(B)-\mu\right]\right\}^{d} } \\
\times & \times\left(\chi_{c} \prod_{k=1}^{d} b_{k}\right)^{d-1},
\end{aligned}
$$

where $\chi_{c}=\theta_{c}$ for $F$ nonarithmetic. Using similar arguments, (3.9) can also be shown to hold for $F$ arithmetic with span $\eta$, by defining $\nu_{c}$ in (3.7) with $y \rightarrow \infty$ over $\eta \mathbb{Z}$ and $\chi_{c}=\eta^{-1}\left(1-\mathrm{e}^{-\eta \theta_{c}}\right)$.

Example 3.3. If $F$ has point mass at 1 then $\sup \{Y(u): Y(u)<0\}=-1$ almost surely, and, by (2.11) and Jensen's inequality,

$$
K=\mathrm{E}\left(\sigma_{d}^{-1}(\{u: Y(u)=0\})\right) \geq\left\{\mathrm{E}\left(\sigma_{d}(\{u: Y(u)=0\})\right)\right\}^{-1} .
$$

Let $U_{d}=\{t:\|t\| \leq 1\}$ be the $d$-dimensional unit ball, and let

$$
C_{d}=\frac{\sigma_{d-1}\left(\partial U_{d}\right)}{\sigma_{d-1}^{d}\left(U_{d-1}\right)}=\frac{d \pi^{d / 2} / \Gamma(d / 2+1)}{\left[\pi^{(d-1) / 2} / \Gamma((d+1) / 2)\right]^{d}} .
$$

Then

$$
\begin{aligned}
\mathrm{E}\left[\sigma_{d}(\{u: Y(u)=0\})\right] & =C_{d} \int_{0}^{\infty} r^{d-1} \sum_{k=0}^{\infty} \mathrm{e}^{-r(1+c)} \frac{c^{k} r^{2 k}}{(k !)^{2}} \mathrm{~d} r \\
& =C_{d} \sum_{k=0}^{\infty} \frac{c^{k} \Gamma(2 k+d)}{(k !)^{2}(1+c)^{2 k+d}} \\
& =\frac{C_{d}}{(1+c)^{d}} f_{d}^{(d-1)} \frac{\sqrt{c}}{(1+c)},
\end{aligned}
$$

where $f_{d}(x)=x^{d} / \sqrt{1-4 x^{2}}$ and $f_{d}^{(d-1)}$ is the $(d-1)$ th derivative of $f_{d}$. A positive lower bound of $K$ follows from (3.10). Similar computations can also be carried out for kernels of other shapes.

Example 3.4. Let $\Omega$ denote the random set of all $u$ for which the sums in (2.3) are sums over an empty set for both $j=1$ and $j=2$. For a more precise definition, let $\left\{\left(t_{i}, y_{i}\right): i \geq 1\right\}$ be a Poisson point process on $\partial B \times \mathbb{R}$ with intensity 1 on $\partial B \times[0, \infty)$ and intensity $M\left(\theta_{c}\right)$ on $\partial B \times(-\infty, 0)$. Then

$$
\Omega=\left\{u: y_{i} \geq n_{t_{i}} \cdot u>0 \text { or } y_{i}<n_{t_{i}} \cdot u<0 \text { for all } i\right\} .
$$


By (2.4), $\Omega \subset\{u: Y(u)=0\}$ and, hence, by (2.11),

$$
K \leq \begin{cases}|\eta| \mathrm{E}\left(\sigma_{d}^{-1}(\Omega)\right) & \text { if } F \text { has point mass at } \eta \\ \chi_{c}^{-1} \mathrm{E}\left(\sigma_{d}^{-1}(\Omega)\right) & \text { otherwise. }\end{cases}
$$

The upper bound of $K$ for $F$ having point mass at $\eta$ uses the property

$$
\sup \{Y(u): Y(u)<0\}=-|\eta| \text { almost surely. }
$$

\section{Proof of Theorem 2.1}

A key idea here is a change of measure argument that allows us to obtain, in Lemma 4.1, below, the tail probability of the maxima over a local domain. To obtain the global probabilities in Theorem 2.1 from these local probabilities, we adapt the Pickands-Quall-Watanabe technique from the Gaussian random field literature. Hence, the characterization of the constant $K$ in Theorem 2.1 bears a striking resemblance to constants seen in the earlier papers on Gaussian random fields though the distribution of $Y(u)$ here is compound Poisson rather than Gaussian. Hogan and Siegmund [12] provided a vital framework for this correspondence.

Lemma 4.1. Let $E_{t, m, \lambda}=\left\{\sup _{t \leq v \leq t+m \lambda^{-1}} S(v+B) \geq \lambda c\right\}$. Define $x_{\lambda}$ as in the statement of Theorem 2.1. Then, for all $t \in D$,

$$
\mathrm{P}_{\lambda}\left(E_{t, m, \lambda}\right)=\mathrm{P}_{\lambda}\left(E_{0, m, \lambda}\right) \sim\left[2 \pi \lambda \sigma_{d}(B) M^{\prime \prime}\left(\theta_{c}\right)\right]^{-1 / 2} \mathrm{e}^{-\lambda I+x_{\lambda}} K_{m} \quad \text { as } \lambda \rightarrow \infty .
$$

Proof. By stationarity, $\mathrm{P}_{\lambda}\left(E_{t, m, \lambda}\right)=\mathrm{P}_{\lambda}\left(E_{0, m, \lambda}\right)$. Let us first consider the case in which $F$ is arithmetic with span 1 . Then

$$
\begin{aligned}
\mathrm{P}_{\lambda}\left(E_{0, m, \lambda}\right)= & \mathrm{P}_{\lambda}\left(\sup _{0 \leq v \leq m \lambda^{-1}} S(v+B) \geq \lambda c\right) \\
= & \mathrm{P}_{\lambda}\{S(B) \geq\lfloor\lambda c\rfloor\} \\
& +\sum_{\ell=1}^{\infty} \mathrm{P}_{\lambda}\left(S(B)=\lfloor\lambda c\rfloor-\ell, \sup _{0 \leq v \leq m \lambda^{-1}}[S(v+B)-S(B)] \geq \ell\right) .
\end{aligned}
$$

Let $\mathrm{Q}_{\lambda}$ be a probability measure under which $\mathcal{X}$ is a nonhomogeneous marked point process with intensity $\lambda M\left(\theta_{c}\right)$ and mark distribution $F_{c}$ inside $B$, and intensity $\lambda$ and mark distribution $F$ outside $B$. Then

$$
\frac{\mathrm{dQ} \lambda}{\mathrm{dP}_{\lambda}}(\mathcal{X})=\exp \left\{\theta_{c} S(B)-\lambda \sigma_{d}(B)\left[M\left(\theta_{c}\right)-1\right]\right\}
$$

Let

$$
B_{a}=\bigcap_{0 \leq v \leq a}(v+B)
$$

Since $S\left(B \backslash B_{m \lambda^{-1}}\right)$ and $\sup _{0 \leq v \leq m \lambda^{-1}}[S(v+B)-S(B)]$ are functions of the marked point 
process outside $B_{m \lambda^{-1}}$ and, hence, independent of $S\left(B_{m \lambda^{-1}}\right)$ under $Q_{\lambda}$, it follows from (4.3) that

$$
\begin{gathered}
\mathrm{P}_{\lambda}\left(S(B)=\lfloor\lambda c\rfloor-\ell, \sup _{0 \leq v \leq m \lambda^{-1}}[S(v+B)-S(B)] \geq \ell\right) \\
=\exp \left\{-\lambda I+x_{\lambda}+\theta_{c} \ell\right\} \mathrm{Q}_{\lambda}\left(S(B)=\lfloor\lambda c\rfloor-\ell, \sup _{0 \leq v \leq m \lambda^{-1}}[S(v+B)-S(B)] \geq \ell\right) \\
=\exp \left\{-\lambda I+x_{\lambda}+\theta_{c} \ell\right\} \sum_{k=0}^{\infty} \mathrm{Q}_{\lambda}\left(S\left(B_{m \lambda^{-1}}\right)=\lfloor\lambda c\rfloor-\ell-k\right) \\
\times \mathrm{Q}_{\lambda}\left(S\left(B \backslash B_{m \lambda^{-1}}\right)=k, \sup _{0 \leq v \leq m \lambda^{-1}}[S(v+B)-S(B)] \geq \ell\right) .
\end{gathered}
$$

It follows from the local central limit theorem that, for each $\ell \in \mathbb{Z}$,

$$
\begin{aligned}
\mathrm{Q}_{\lambda}\left(S\left(B_{m \lambda^{-1}}\right)=\lfloor\lambda c\rfloor-\ell-k\right) & \leq[1+o(1)] \mathrm{Q}_{\lambda}(S(B)=\lfloor\lambda c\rfloor-\ell) \\
& \sim\left[2 \pi \lambda \sigma_{d}(B) M^{\prime \prime}\left(\theta_{c}\right)\right]^{-1 / 2} \quad \text { as } \lambda \rightarrow \infty,
\end{aligned}
$$

uniformly over $k \geq 0$, with ' $\leq$ ' replaced by ' $=$ ' if we look at (4.5) with $k$ fixed. Hence, by (4.4) and (4.5),

$$
\begin{gathered}
\mathrm{P}_{\lambda}\left(S(B)=\lfloor\lambda c\rfloor-\ell, \sup _{0 \leq v \leq m \lambda^{-1}}[S(v+B)-S(B)] \geq \ell\right) \\
\sim\left[2 \pi \lambda \sigma_{d}(B) M^{\prime \prime}\left(\theta_{c}\right)\right]^{-1 / 2} \exp \left\{-\lambda I+x_{\lambda}+\theta_{c} \ell\right\} \\
\times \mathrm{Q}_{\lambda}\left(\sup _{0 \leq v \leq m \lambda^{-1}}[S(v+B)-S(B)] \geq \ell\right) .
\end{gathered}
$$

By (4.6) and the weak convergence of $\left\{S\left(\lambda^{-1} u+B\right)-S(B): 0 \leq u \leq m\right\}$ under $\mathrm{Q}_{\lambda}$ to $\{Y(u): 0 \leq u \leq m\}$ as $\lambda \rightarrow \infty$ (see (2.3) and (2.4)),

$$
\begin{aligned}
& \sum_{\ell=1}^{\infty} \mathrm{P}_{\lambda}\left(S(B)=\lfloor\lambda c\rfloor-\ell, \sup _{0 \leq v \leq m \lambda^{-1}}[S(v+B)-S(B)] \geq \ell\right) \\
& \quad \sim \mathrm{e}^{-\lambda I+x_{\lambda}}\left[2 \pi \lambda \sigma_{d}(B) M^{\prime \prime}\left(\theta_{c}\right)\right]^{-1 / 2} \sum_{\ell=1}^{\infty} \mathrm{e}^{\theta_{c} \ell} \mathrm{P}\left(\sup _{0 \leq u \leq m} Y(u) \geq \ell\right) .
\end{aligned}
$$

By a similar application of (4.3) and (4.5),

$$
\mathrm{P}_{\lambda}(S(B) \geq\lfloor\lambda c\rfloor) \sim\left[2 \pi \lambda \sigma_{d}(B) M^{\prime \prime}\left(\theta_{c}\right)\right]^{-1 / 2} \mathrm{e}^{-\lambda I+x_{\lambda}} \sum_{\ell=-\infty}^{0} \mathrm{e}^{\theta_{c} \ell} .
$$

Substitution of (4.7) and (4.8) into (4.2) then proves Lemma 4.1 when $F$ is arithmetic with span 1. For $F$ arithmetic with span $\eta$, we prove Lemma 4.1 by replacing the sums in (4.2), (4.4), (4.7), and (4.8) by $\sum_{\ell \in \eta \mathbb{Z}^{+}}, \sum_{k \geq 0, k \in \eta \mathbb{Z}}$, or $\sum_{\ell \leq 0, \ell \in \eta \mathbb{Z}}$. For nonarithmetic $F$, the sums are replaced by corresponding integrals. The details are similar to the arguments provided above and shall be omitted. 
We now proceed to the proof of Theorem 2.1. Let $J=[0,1]^{d}$,

$$
\underline{C}_{a}=\left\{k \in(a \mathbb{Z})^{d}: k+a J \subset D\right\}, \quad \text { and } \quad \bar{C}_{a}=\left\{k \in(a \mathbb{Z})^{d}:(k+a J) \cap D \neq \varnothing\right\} .
$$

Then $\left\{k+a J: k \in \underline{C}_{a}\right\}$ and $\left\{k+a J: k \in \bar{C}_{a}\right\}$ are lower and upper coverings of $D$, respectively, by cubes of length $a$. We shall show via Lemmas 4.2 and 4.3, below, that

$$
\lim _{m \rightarrow \infty} \limsup _{\lambda \rightarrow \infty}\left[\frac{\sum_{u \in \underline{C}_{m \lambda^{-1}}} \mathrm{P}_{\lambda}\left(\bigcup_{\left\{w \in \underline{C}_{m \lambda^{-1}}: w \neq u\right\}}\left(E_{u, m, \lambda} \cap E_{w, m, \lambda}\right)\right)}{\lambda^{d-1 / 2} \mathrm{e}^{-\lambda I}}\right]=0 .
$$

The proofs of Lemmas 4.2, 4.3, and (4.9) will be given in the appendices. Let $f(\lambda)=$ $\left[2 \pi \sigma_{d}(B) M^{\prime \prime}\left(\theta_{c}\right)\right]^{-1 / 2} \lambda^{d-1 / 2} \mathrm{e}^{-\lambda I+x_{\lambda}}$. Then, by Lemma 4.1 ,

$$
\mathrm{P}_{\lambda}\left(E_{u, m, \lambda}\right) \sim \frac{K_{m} f(\lambda)}{\lambda^{d}} \quad \text { as } \lambda \rightarrow \infty .
$$

Given $\varepsilon>0$, let $m_{\varepsilon}$ be large enough such that, for all $m \geq m_{\varepsilon}$, the expression in the square brackets on the left-hand side of (4.9) does not exceed $\varepsilon$ for all large $\lambda$. Then, by (4.10), for all $m \geq m_{\varepsilon}$,

$$
\begin{aligned}
\liminf _{\lambda \rightarrow \infty}\left[\lambda^{-d}\left(\# \underline{C}_{m / \lambda}\right) K_{m}-\frac{\varepsilon \lambda^{d-1 / 2} \mathrm{e}^{-\lambda I}}{f(\lambda)}\right] & \leq \liminf _{\lambda \rightarrow \infty}\left[\frac{p_{\lambda}}{f(\lambda)}\right] \\
& \leq \limsup _{\lambda \rightarrow \infty}\left[\frac{p_{\lambda}}{f(\lambda)}\right] \\
& \leq \limsup _{\lambda \rightarrow \infty}\left[\lambda^{-d}\left(\# \bar{C}_{m / \lambda}\right) K_{m}\right] .
\end{aligned}
$$

Since $D$ is Jordan measurable,

$$
\# \underline{C}_{a} \sim \# \bar{C}_{a} \sim a^{-d} \sigma_{d}(D) \quad \text { as } a \rightarrow 0 .
$$

Noting that $\lim _{\inf }{ }_{\lambda \rightarrow \infty}\left[p_{\lambda} / f(\lambda)\right]$ and $\lim \sup _{\lambda \rightarrow \infty}\left[p_{\lambda} / f(\lambda)\right]$ are fixed real numbers and $x_{\lambda}$ is bounded, it follows from (4.11) and (4.12) that $m^{-d} K_{m}$ is Cauchy. Hence,

$$
K=\lim _{m \rightarrow \infty} m^{-d} K_{m}
$$

exists and (2.8) follows from (4.11).

We will now state Lemmas 4.2 and 4.3 before proving Theorem 2.1. To avoid repetitive arguments, we will state and prove all subsequent results in this section and in the appendices assuming that $F$ is arithmetic with span 1 . The modifications required to extend these results to arbitrary $F$ are straightforward and will not be discussed.

Lemma 4.2. We have

$$
\lim _{r \rightarrow \infty} \limsup _{\lambda \rightarrow \infty}\left[\frac{\mathrm{P}_{\lambda}\left(S(B)<\lfloor\lambda c\rfloor-r, \sup _{0 \leq v \leq \lambda^{-1}} S(v+B) \geq\lfloor\lambda c\rfloor\right)}{\lambda^{-1 / 2} \mathrm{e}^{-\lambda I}}\right]=0 .
$$

Lemma 4.3. Let $r \geq 0$ and $L>0$ be given. Then

$\lim _{k \rightarrow \infty} \limsup _{\lambda \rightarrow \infty}\left[\frac{\sum_{\left\{v \in\left(\lambda^{-1} \mathbb{Z}\right)^{d}: k \lambda^{-1} \leq\|v\|_{\infty} \leq L\right\}} \mathrm{P}_{\lambda}(S(B) \geq\lfloor\lambda c\rfloor-r, S(v+B) \geq\lfloor\lambda c\rfloor-r)}{\lambda^{-1 / 2} \mathrm{e}^{-\lambda I}}\right]=0$. 
Proof of Theorem 2.1. By the arguments in (4.9)-(4.12), it remains for us to show that $K$ is positive and finite. By Lemma 4.2, there exists $r$ large enough such that

$$
\mathrm{P}_{\lambda}\left(S(B)<\lfloor\lambda c\rfloor-r, \sup _{0 \leq v \leq \lambda^{-1}} S(v+B) \geq\lfloor\lambda c\rfloor\right) \leq \lambda^{-1 / 2} \mathrm{e}^{-\lambda I} \quad \text { for all large } \lambda .
$$

Moreover, by a modification of (4.8) (with $\sum_{\ell=-\infty}^{r}$ in place of $\sum_{\ell=-\infty}^{0}$ ),

$$
\mathrm{P}_{\lambda}(S(B) \geq\lfloor\lambda c\rfloor-r)=O\left(\lambda^{-1 / 2} \mathrm{e}^{-\lambda I}\right) .
$$

By adding up (4.13) and (4.14), and applying Lemma 4.1, we show that $K_{1}<\infty$. Then, by (2.8), (4.11), and (4.12), $K \leq K_{1}<\infty$.

Next, select $\varepsilon$ small enough so that $\delta=\left[2 \pi \sigma_{d}(B) M^{\prime \prime}\left(\theta_{c}\right)\right]^{-1 / 2} \sum_{\ell=-\infty}^{0} \mathrm{e}^{\theta_{c} \ell}-\varepsilon>0$. By Lemma 4.3 with $r=0$ and $L>\sup _{x, y \in D}\|x-y\|_{\infty}$, there exists $k$ large enough such that

$$
\sum_{\left\{v \in\left(k \lambda^{-1} \mathbb{Z}\right)^{d}: 0<\|v\|_{\infty} \leq L\right\}} \mathrm{P}_{\lambda}(S(B) \geq\lfloor\lambda c\rfloor, S(v+B) \geq\lfloor\lambda c\rfloor) \leq \varepsilon \lambda^{-1 / 2} \mathrm{e}^{-\lambda I}
$$

for all large $\lambda$. Then, by stationarity, (4.8), and (4.15), noting that $x_{\lambda} \geq 0$,

$$
\begin{aligned}
\mathrm{P}_{\lambda}\left(E_{\mathbf{0}, m, \lambda}\right) \geq & \mathrm{P}_{\lambda}\left(S(u+B) \geq\lfloor\lambda c\rfloor \text { for some } u \in\left(k \lambda^{-1} \mathbb{Z}\right)^{d}, 0 \leq u \leq m \lambda^{-1}\right) \\
\geq & \sum_{\left\{u \in\left(k \lambda^{-1} \mathbb{Z}\right)^{d}: 0 \leq u \leq m \lambda^{-1}\right\}}\left(\mathrm{P}_{\lambda}(S(u+B) \geq\lfloor\lambda c\rfloor)\right. \\
& \left.-\sum_{\left\{w \in\left(k \lambda^{-1} \mathbb{Z}\right)^{d}: 0<\|w-u\|_{\infty} \leq L\right\}} \mathrm{P}\{S(u+B) \geq\lfloor\lambda c\rfloor, S(w+B) \geq\lfloor\lambda c\rfloor\}\right) \\
\geq & \left(\frac{m}{k}\right)^{d} \delta \lambda^{-1 / 2} \mathrm{e}^{-\lambda I} \quad \text { for all large } \lambda,
\end{aligned}
$$

and, by letting $m \rightarrow \infty$ with $k$ fixed, it follows from (2.7), (4.1), and $x_{\lambda}$ bounded that $K>0$.

\section{Proof of Theorem 2.2}

The key trick here is a telescoping sum of (5.3) or (5.5), below, which removes the dependence of the representation of $K$ on the supremum of $Y$. To simplify the notation, we shall first consider $F$ having point mass at 1 , select $\lambda$ such that $x_{\lambda}=0$ (i.e. $\lambda c \in \mathbb{Z}$ ), and write $S(v)$ in place of $S(v+B)$. Let

$p_{c}(t, k, \mathrm{~d} w)=\mathrm{P}_{\lambda}\left(\sup _{v \in D} S(v)=\lambda c, S(t)=\lambda c-k, \sigma_{d}(\{v \in D: S(v)=\lambda c-k\}) \in \lambda^{-d} \mathrm{~d} w\right)$.

By the change of measure argument in the proof of Lemma 4.1, in particular (4.4) and (4.5), and Lemmas 4.2 and 4.3, for any integer $k \geq 0, t$ lying in the interior of $D$ and $\mathrm{d} w \in(0, \infty)$,

$$
\begin{aligned}
& p_{c}(t, k, d w)= \mathrm{e}^{-\lambda I+\theta_{c} k} \mathrm{Q}_{\lambda}(S(t)=\lambda c-k) \mathrm{Q}_{\lambda}\left(\sup _{v \in D}[S(v)-S(t)]=k,\right. \\
&\left.\sigma_{d}(\{v \in D:[S(v)-S(t)]=0\}) \in \lambda^{-d} \mathrm{~d} w \mid S(t)=\lambda c-k\right) \\
& \sim\left[2 \pi \lambda \sigma_{d}(B) M^{\prime \prime}\left(\theta_{c}\right)\right]^{-1 / 2} \mathrm{e}^{-\lambda I+\theta_{c} k} \\
& \\
& \quad \times \mathrm{P}\left(\sup _{u \in \mathbb{R}^{d}} Y(u)=k, \sigma_{d}(\{u: Y(u)=0\}) \in \mathrm{d} w\right) .
\end{aligned}
$$


The second relation in (5.2) follows from the weak convergence of $\left\{S\left(\lambda^{-1} u+t\right)-S(t): 0 \leq\right.$ $u \leq m\}$, under $\mathrm{Q}_{\lambda}$ and with conditioning on $S(t)=\lambda c-k$, to $\{Y(u): 0 \leq u \leq m\}$. Multiplying $p_{c}(t, k, \mathrm{~d} w)$ by $\lambda^{d}\left(\mathrm{e}^{-\theta_{c} k}-\mathrm{e}^{-\theta_{c}(k+1)}\right) / w$ and integrating over $t \in D$ and $w>0$, we obtain, from (5.1) and (5.2),

$$
\begin{aligned}
\left(\mathrm{e}^{-\theta_{c} k}-\mathrm{e}^{-\theta_{c}(k+1)}\right) \mathrm{P}_{\lambda}\left(\sup _{v \in D} S(v)=\lambda c, S(t)=\lambda c-k \text { for some } t \in D\right) \\
=\lambda^{d}\left(\mathrm{e}^{-\theta_{c} k}-\mathrm{e}^{-\theta_{c}(k+1)}\right) \int_{D} \int_{0}^{\infty} \frac{p_{c}(t, k, \mathrm{~d} w)}{w} \mathrm{~d} t \\
\sim\left[2 \pi \sigma_{d}(B) M^{\prime \prime}\left(\theta_{c}\right)\right]^{-1 / 2} \mathrm{e}^{-\lambda I} \lambda^{d-1 / 2} \sigma_{d}(D)\left(1-\mathrm{e}^{-\theta_{c}}\right) \\
\quad \times \mathrm{E}\left(\sigma_{d}^{-1}(\{u: Y(u)=0\}) \mathbf{1}_{\left\{\sup _{u \in \mathbb{R}^{d}} Y(u)=k\right\}}\right) .
\end{aligned}
$$

We then sum up over $0 \leq k \leq k_{0}$, let $\lambda \rightarrow \infty$ followed by $k_{0} \rightarrow \infty$, and compare against the asymptotic relation

$$
\mathrm{P}_{\lambda}\left(\sup _{v \in D} S(v)=\lambda c\right) \sim\left[2 \pi \sigma_{d}(B) M^{\prime \prime}\left(\theta_{c}\right)\right]^{-1 / 2}\left(1-\mathrm{e}^{-\theta_{c}}\right) \mathrm{e}^{-\lambda I} \lambda^{d-1 / 2} \sigma_{d}(D) K,
$$

which follows from a straightforward modification of Theorem 2.1, to obtain the expression $K=\mathrm{E}\left(\sigma_{d}^{-1}(\{u: Y(u)=0\})\right)$.

Let us next consider more generally $F$ arithmetic with span 1 . Let $k_{0}>0$, and when the event $\left\{\sup _{v \in D} S(v)=\lambda c\right\}$ occurs, let $\left\{r_{i}: 1 \leq i \leq I\right\}$ be a monotone rearrangement of $\left\{\lambda c-S(t): t \in D, 0 \leq \lambda c-S(t) \leq k_{0}\right\}$. In other words, $0=r_{0}<r_{1}<\cdots<r_{I} \leq k_{0}$. Define, for $0 \leq k<\ell \leq k_{0}$,

$$
\begin{aligned}
& p_{c}(t, k, \ell, \mathrm{d} w)=\mathrm{P}\left(\sup _{v \in D} S(v)=\lambda c, \lambda c-S(t)=k=r_{i}\right. \text { and } \\
& \qquad \begin{array}{l}
\left.\ell=r_{i+1} \text { for some } i, \sigma_{d}(\{v: S(v)=\lambda c-k\}) \in \lambda^{-d} \mathrm{~d} w\right) .
\end{array}
\end{aligned}
$$

Then, by the arguments in (5.2) and (5.3),

$$
\begin{aligned}
\left(\mathrm{e}^{-\theta_{c} k}-\mathrm{e}^{-\theta_{c} \ell}\right) \mathrm{P}_{\lambda}\left(\sup _{v \in D} S(v)=\lambda c, r_{i}=k, r_{i+1}=\ell \text { for some } i\right) \\
=\lambda^{d}\left(\mathrm{e}^{-\theta_{c} k}-\mathrm{e}^{-\theta_{c} \ell}\right) \int_{D} \int_{0}^{\infty} \frac{p_{c}(t, k, \ell, \mathrm{d} w)}{w} \mathrm{~d} t \\
\sim\left[2 \pi \sigma_{d}(B) M^{\prime \prime}\left(\theta_{c}\right)\right]^{-1 / 2} \mathrm{e}^{-\lambda I} \lambda^{d-1 / 2} \sigma_{d}(D)\left(1-\mathrm{e}^{-\theta_{c}(\ell-k)}\right) \\
\quad \times \mathrm{E}\left(\sigma_{d}^{-1}(\{u: Y(u)=0\}) \mathbf{1}_{\left\{\sup _{u \in \mathbb{R}^{d}} Y(u)=k, \sup _{u \in \mathbb{R}^{d}: Y(u)<0} Y(u)=k-\ell\right\}}\right) .
\end{aligned}
$$

We then sum up (5.5) over all integers $0 \leq k<\ell \leq k_{0}$, let $\lambda \rightarrow \infty$ followed by $k_{0} \rightarrow \infty$, and compare against (5.4) to obtain the more general expression (2.11). For $F$ arithmetic with span $\eta$, the arguments are similar, while for nonarithmetic $F$, we need to partition the range of $S(t), t \in D$, into intervals of length $\varepsilon$, then let $\varepsilon \rightarrow 0$. The details are omitted.

\section{Extension of representation to Gaussian random fields}

Let $X(t), t \in \mathbb{R}^{d}$, be a stationary, mean zero, continuous Gaussian random field satisfying

$$
\mathrm{E}(X(t) X(t+s)) \sim 1-a\|s\|^{\alpha} \quad \text { as }\|s\| \rightarrow 0
$$


for some $0<\alpha \leq 2$ and $a>0$. Let $\Delta_{c}=\left(c^{2} a\right)^{-1 / \alpha}$. It was shown in [6], [16], and [18] that

$$
\mathrm{P}\left(\sup _{t \in D} X(t) \geq c\right) \sim(2 \pi)^{-1 / 2} c^{-1} \mathrm{e}^{-c^{2} / 2} \Delta_{c}^{-d} \sigma_{d}(D) \tilde{K} \quad \text { as } c \rightarrow \infty
$$

for some $\tilde{K}>0$, which is expressed in terms of a continuous Gaussian random field $\tilde{Y}$ satisfying

$$
\mathrm{E}(\tilde{Y}(u))=-\|u\|^{\alpha}, \quad \operatorname{cov}(\tilde{Y}(u), \tilde{Y}(v))=\|u\|^{\alpha}+\|v\|^{\alpha}-\|u-v\|^{\alpha} .
$$

In particular,

$$
\widetilde{K}=\lim _{m \rightarrow \infty} m^{-d} \int_{0}^{\infty} \mathrm{e}^{y} \mathrm{P}\left(\sup _{0 \leq u \leq m} \tilde{Y}(u) \geq y\right) \mathrm{d} y .
$$

The key argument in the proof of (6.1) is the weak convergence

$$
\left\{c\left[X\left(t+\Delta_{c} u\right)-X(t)\right]: 0 \leq u \leq m\right\} \Rightarrow\{\tilde{Y}(u): 0 \leq u \leq m\} \text { for any } m>0,
$$

conditioned on $X(t)=c-y / c$ for some $y>0$. In Theorem 6.1, below, we apply the approach of Section 5 to obtain a different representation of $\widetilde{K}$, one that involves only the occupation measure of $\widetilde{Y}$ near 0 .

Theorem 6.1. We have $\widetilde{K}=\lim _{\xi \rightarrow 0} \int_{0}^{\xi} \mathrm{E}\left(\sigma_{d}^{-1}(\{u:-b<\tilde{Y}(u) \leq \xi-b\})\right) \mathrm{d} b$.

Example 6.1. By Theorem 6.1 and Jensen's inequality,

$$
\widetilde{K} \geq \limsup _{\xi \rightarrow 0} \int_{0}^{\xi}\left\{\mathrm{E}\left(\sigma_{d}(\{u:-b<\tilde{Y}(u) \leq \xi-b\})\right)\right\}^{-1} \mathrm{~d} b=\left(\int_{\mathbb{R}^{d}} f_{t}(0) \mathrm{d} t\right)^{-1},
$$

where $f_{t}$ denotes the normal density of $\tilde{Y}(t)$, with means and variances given in (6.2). Let $U_{d}=\{t:\|t\| \leq 1\}$. Then, by (6.4),

$$
\begin{aligned}
\widetilde{K} & \geq\left(\sigma_{d-1}\left(\partial U_{d}\right) \int_{0}^{\infty} r^{d-1}\left(4 \pi r^{\alpha}\right)^{-1 / 2} \exp \left\{-\frac{r^{2 \alpha}}{4 r^{\alpha}}\right\} \mathrm{d} r\right)^{-1} \\
& =d^{-1} \pi^{(1-d) / 2} 4^{1-d / \alpha} \alpha \Gamma\left(\frac{d}{2}+1\right) / \Gamma\left(\frac{d}{\alpha}-\frac{1}{2}\right) .
\end{aligned}
$$

In the case $\alpha=2, \widetilde{Y}$ has a simple characterization from which $\widetilde{K}=\pi^{-d / 2}$ can be computed. For $d=2$, the second line of $(6.5)$ is $\pi^{-1}(=\widetilde{K})$ and, for $d=3$, it is $(4 \sqrt{\pi})^{-1}$.

It is quite straightforward to improve upon (6.5). Consider $0<\alpha<2$ and select $t \neq 0$. Let $f_{s \mid t}(\cdot \mid y)$ denote the density of $\widetilde{Y}(s)$ conditioned on $\widetilde{Y}(t)=y$. Then, by a more refined application of Jensen's inequality,

$$
\widetilde{K} \geq \int_{-\infty}^{\infty}\left(\int_{\mathbb{R}^{d}} f_{s \mid t}(0 \mid y) \mathrm{d} s\right)^{-1} f_{t}(y) \mathrm{d} y .
$$

In principle, we can get even sharper computable lower bounds by conditioning on two or more $t \mathrm{~s}$.

Proof of Theorem 6.1. Let $\xi>0,0 \leq v<\xi, X_{\text {sup }}=\sup _{t \in D} X(t)$, and $Y_{\text {sup }}=$ $\sup _{u \in \mathbb{R}^{d}} \tilde{Y}(u)$. Let $k$ be a nonnegative integer, $I_{k}=\left(X_{\text {sup }}+[v-(k+1) \xi] / c, X_{\text {sup }}+(v-k \xi) / c\right]$, and $J_{k}=[k \xi-v,(k+1) \xi-v)$. Define

$$
g_{c, \xi, v}(t, k, \mathrm{~d} w)=\mathrm{P}\left(X_{\text {sup }} \geq c, X(t) \in I_{k}, \sigma_{d}\left(\left\{u \in D: X(u) \in I_{k}\right\}\right) \in \Delta_{c}^{d} \mathrm{~d} w\right) .
$$


By (6.3), for any $t$ lying in the interior of $D$ and $\mathrm{d} w \in(0, \infty)$,

$$
\begin{aligned}
& g_{c, \xi, v}(t, k, \mathrm{~d} w) \\
& \begin{array}{r}
\sim(2 \pi)^{-1 / 2} c^{-1} \mathrm{e}^{-c^{2} / 2} \int_{-\infty}^{\infty} \mathrm{e}^{y} \mathrm{P}\left(X_{\text {sup }} \geq c, X(t) \in I_{k},\right. \\
\left.\sigma_{d}\left(\left\{u \in D: X(u) \in I_{k}\right\}\right) \in \Delta_{c}^{d} \mathrm{~d} w \mid X(t)=c-\frac{y}{c}\right) \mathrm{d} y
\end{array} \\
& \sim(2 \pi)^{-1 / 2} c^{-1} \mathrm{e}^{-c^{2} / 2}\left\{\left(\int_{-\infty}^{k \xi-v} \mathrm{e}^{y} \mathrm{~d} y\right) \mathrm{P}\left(Y_{\text {sup }} \in J_{k}, \sigma_{d}\left(\left\{u: Y_{\text {sup }}-\tilde{Y}(u) \in J_{k}\right\}\right) \in \mathrm{d} w\right)\right. \\
& +\int_{k \xi-v}^{(k+1) \xi-v} \mathrm{e}^{y} \mathrm{P}\left(y \leq Y_{\text {sup }}<(k+1) \xi-v,\right. \\
& \left.\left.\sigma_{d}\left(\left\{u: Y_{\text {sup }}-\tilde{Y}(u) \in J_{k}\right\}\right) \in \mathrm{d} w\right) \mathrm{~d} y\right\} .(6.6)
\end{aligned}
$$

Multiply (6.6) by $\left(\mathrm{e}^{-k \xi}-\mathrm{e}^{-(k+1) \xi}\right) /\left(\Delta_{c}^{d} w\right)$, integrate over $t \in D$ and $w>0$, then add over $0 \leq k \leq k_{0}$ for some positive integer $k_{0}>0$. We obtain

$$
\begin{aligned}
& \sum_{k=0}^{k_{0}}\left(\mathrm{e}^{-k \xi}-\mathrm{e}^{-(k+1) \xi}\right) \mathrm{P}\left(X_{\text {sup }} \geq c, X(t) \in I_{k} \text { for some } t \in D\right) \\
& =\Delta_{c}^{-d} \sum_{k=0}^{k_{0}}\left(\mathrm{e}^{-k \xi}-\mathrm{e}^{-(k+1) \xi}\right) \int_{D} \int_{0}^{\infty} \frac{g_{c, \xi, v}(t, k, \mathrm{~d} w)}{w} \mathrm{~d} t \\
& \sim(2 \pi)^{-1 / 2} c^{-1} \mathrm{e}^{-c^{2} / 2} \Delta_{c}^{-d} \sigma_{d}(D) \\
& \quad \times\left\{\left(\mathrm{e}^{-v}-\mathrm{e}^{-v-\xi}\right) \sum_{k=0}^{k_{0}} \mathrm{E}\left(\mathbf{1}_{\left\{Y_{\text {sup }} \in J_{k}\right\}} \sigma_{d}^{-1}\left(\left\{u: Y_{\text {sup }}-\tilde{Y}(u) \in J_{k}\right\}\right)\right)+o_{\xi}(1)\right\}
\end{aligned}
$$

where $o_{\xi}(1) \rightarrow 0$ as $\xi \rightarrow 0$. Since $Y_{\text {sup }} \in J_{k}$ is equivalent to $k=\left\lfloor\left(Y_{\text {sup }}+v\right) / \xi\right\rfloor$, by (6.1) and (6.7), and letting $k_{0} \rightarrow \infty$,

$$
\begin{gathered}
\tilde{K}=\lim _{\xi \rightarrow 0} \int_{0}^{\xi} \mathrm{E}\left(\sigma _ { d } ^ { - 1 } \left(\left\{u: Y_{\text {sup }}-\left(\xi\left\lfloor\frac{Y_{\text {sup }}+v}{\xi}\right\rfloor-v-\xi\right)<\widetilde{Y}(u)\right.\right.\right. \\
\left.\left.\left.\leq Y_{\text {sup }}-\left(\xi\left\lfloor\frac{Y_{\text {sup }}+v}{\xi}\right\rfloor-v\right)\right\}\right)\right) \mathrm{d} v
\end{gathered}
$$

and Theorem 6.1 is shown.

\section{Appendix A. Proof of Lemma 4.2}

By (4.6) with $m=1$ and the weak convergence of $S\left(\lambda^{-1} u+B\right)-S(B)$ to $Y(u)$ under $\mathrm{Q}_{\lambda}$, as $\lambda \rightarrow \infty$,

$$
\begin{aligned}
& \mathrm{P}_{\lambda}\left(S(B)<\lfloor\lambda c\rfloor-r, \sup _{0 \leq v \leq \lambda^{-1}} S(v+B) \geq\lfloor\lambda c\rfloor\right) \\
& \quad \sim\left[2 \pi \lambda \sigma_{d}(B) M^{\prime \prime}\left(\theta_{c}\right)\right]^{-1 / 2} \mathrm{e}^{-\lambda I+x_{\lambda}} \sum_{\ell=r+1}^{\infty} \mathrm{e}^{\theta_{c} \ell} \mathrm{P}\left(\sup _{0 \leq u \leq 1} Y(u) \geq \ell\right) .
\end{aligned}
$$


Let $x^{+}=\max \{x, 0\}$ and $x^{-}=\max \{-x, 0\}$. It follows from (2.3) and (2.4) that

$$
\sup _{0 \leq u \leq 1} Y(u) \leq Z^{*}=\sum_{\left\{i: v_{i}^{(1)} \in A^{(1)}\right\}}\left[Z_{i}^{(1)}\right]^{+}+\sum_{\left\{i: v_{i}^{(2)} \in A^{(2)}\right\}}\left[Z_{i}^{(2)}\right]^{-},
$$

where $A^{(1)}=\partial B \times\left[0, d^{1 / 2}\right)$ and $A^{(2)}=\partial B \times\left[-d^{-1 / 2}, 0\right)$. We can also express $Z^{*}=$ $\sum_{j=1}^{N} V_{j}$, where $N$ is a Poisson random variable with mean $\kappa=1-F(0)+M\left(\theta_{c}\right) F_{c}(0)$ and $V_{1}, V_{2}, \ldots$ are i.i.d. random variables independent of $N$ with $g=\mathrm{E}\left(\mathrm{e}^{\tilde{\theta} V_{1}}\right)<\infty$ for some $\tilde{\theta}>\theta_{c}$. Since $\mathrm{E}\left(\mathrm{e}^{\tilde{\theta} Z^{*}}\right)=\mathrm{e}^{\kappa(g-1)}$, it follows from Markov's inequality that $\mathrm{P}\left(Z^{*} \geq \ell\right) \leq$ $\mathrm{e}^{\kappa(g-1)-\tilde{\theta} \ell}$. Hence, by (A.2),

$$
\sum_{\ell=r+1}^{\infty} \mathrm{e}^{\theta_{c} \ell} \mathrm{P}_{\lambda}\left(\sup _{0 \leq u \leq 1} Y(u) \geq \ell\right)=O\left(\exp \left\{\kappa(g-1)-\left(\tilde{\theta}-\theta_{c}\right) r\right\}\right) .
$$

Since $x_{\lambda}$ is bounded, Lemma 4.2 follows from (A.1) and (A.3).

\section{Appendix B. Proof of Lemma 4.3}

Let $\widetilde{\mathrm{Q}}_{\lambda}\left(=\widetilde{\mathrm{Q}}_{\lambda, v}\right)$ be the probability measure under which the marked point process $\mathcal{X}$ has intensity $\lambda M\left(\theta_{c}\right)$ on $B_{1}=B \cap(v+B)$, intensity $\lambda M\left(\theta_{c} / 2\right)$ on $B_{2}=\{B \backslash(v+B)\} \cup\{(v+B) \backslash B\}$, and intensity $\lambda$ elsewhere on $\mathbb{R}^{d}$. We also require that, under $\widetilde{\mathrm{Q}}_{\lambda}$, the marks have distribution $F_{c}$ on $B_{1}$, distribution $\widetilde{F}$ satisfying $\widetilde{F}(\mathrm{~d} x)=\mathrm{e}^{\theta_{c} x / 2} F(\mathrm{~d} x) / M\left(\theta_{c} / 2\right)$ on $B_{2}$, and $F$ elsewhere on $\mathbb{R}^{d}$. Then

$$
\begin{aligned}
\frac{\mathrm{d} \widetilde{\mathrm{Q}}_{\lambda}}{\mathrm{dP}_{\lambda}}(\mathcal{X})= & \left(\prod_{\left\{i: t_{i} \in B_{1}\right\}} \exp \left\{\theta_{c} X_{i}\right\}\right) \exp \left\{-\lambda \sigma_{d}\left(B_{1}\right)\left[M\left(\theta_{c}\right)-1\right]\right\} \\
& \times\left(\prod_{\left\{i: t_{i} \in B_{2}\right\}} \exp \left\{\frac{\theta_{c} X_{i}}{2}\right\}\right) \exp \left\{-\lambda \sigma_{d}\left(B_{2}\right)\left[M\left(\frac{\theta_{c}}{2}\right)-1\right]\right\} .
\end{aligned}
$$

Since $M$ is convex and $M(0)=1, \zeta=\left[M\left(\theta_{c}\right)-1\right]-2\left[M\left(\theta_{c} / 2\right)-1\right]>0$. We can thus express (B.1) as

$$
\begin{aligned}
\frac{\mathrm{d} \widetilde{\mathrm{Q}}_{\lambda}}{\mathrm{dP}_{\lambda}}(\mathcal{X})=\exp \left\{\frac{\theta_{c}}{2}[S(B)+S(v+B)]-\frac{\lambda\left[\sigma_{d}(B)+\sigma_{d}(v+B)\right]\left[M\left(\theta_{c}\right)-1\right]}{2}\right. \\
\left.+\frac{\lambda \zeta \sigma_{d}\left(B_{2}\right)}{2}\right\},
\end{aligned}
$$

and it follows from (2.5) and an analogue of (4.5) that

$$
\begin{aligned}
\mathrm{P}_{\lambda}(S(B) \geq\lfloor\lambda c\rfloor-r, S(v+B) \geq\lfloor\lambda c\rfloor-r) \\
\quad \leq \mathrm{P}_{\lambda}(S(B)+S(v+B) \geq 2(\lfloor\lambda c\rfloor-r)) \\
\quad=\mathrm{E}_{\tilde{Q}_{\lambda}}\left(\frac{\mathrm{dP}_{\lambda}}{\mathrm{d} \widetilde{\mathrm{Q}}_{\lambda}} \mathbf{1}_{\{S(B)+S(v+B) \geq 2(\lfloor\lambda c\rfloor-r)\})}\right) \\
\quad=O\left(\exp \left\{-\lambda I-\lambda \zeta \sigma_{d}(B \backslash(v+B))\right\} \lambda^{-1 / 2}\right) .
\end{aligned}
$$

Let $\|e\|=1$, and let $\Pi_{e}=\{b-(e \cdot b) e: b \in B\}$ be the projected surface of $B$ on a $(d-1)$ dimensional hyperplane orthogonal to $e$. Then $\beta=\inf _{\|e\|=1} \sigma_{d-1}\left(\Pi_{e}\right)>0$. Hence, there 
exists $\varepsilon>0$ such that

$$
\sigma_{d}(B \backslash(v+B)) \geq \frac{\|v\| \beta}{2} \geq \frac{\|v\|_{\infty} \beta}{2} \text { for all }\|v\|_{\infty} \leq \varepsilon .
$$

By (B.2) and (B.3), it follows that

$$
\begin{aligned}
& \sum_{\left\{v \in\left(\lambda^{-1} \mathbb{Z}\right)^{d}: k \lambda^{-1} \leq\|v\|_{\infty} \leq \varepsilon\right\}} \mathrm{P}_{\lambda}(S(B) \geq\lfloor\lambda c\rfloor-r, S(v+B) \geq\lfloor\lambda c\rfloor-r) \\
& =O\left(\lambda^{-1 / 2} \mathrm{e}^{-\lambda I} \sum_{\ell \geq k} \ell^{d-1} \mathrm{e}^{-\zeta \ell \beta / 2}\right) \\
& =O\left(\lambda^{-1 / 2} \mathrm{e}^{-\lambda I} k^{d-1} \mathrm{e}^{-\zeta k \beta / 2}\right) .
\end{aligned}
$$

Since $\alpha=\inf _{\|v\|_{\infty}>\varepsilon} \sigma_{d}(B \backslash(v+B))>0$, it also follows from (B.2) that

$$
\sum_{\left\{v \in\left(\lambda^{-1} \mathbb{Z}\right)^{d}: \varepsilon<\|v\|_{\infty} \leq L\right\}} \mathrm{P}_{\lambda}(S(B) \geq\lfloor\lambda c\rfloor-r, S(v+B) \geq\lfloor\lambda c\rfloor-r)=O\left(\lambda^{d-1 / 2} \mathrm{e}^{-\lambda I-\lambda \zeta \alpha}\right) .
$$

Lemma 4.3 then follows from combining (B.4) and (B.5).

\section{Appendix C. Proof of (4.9)}

Let $\varepsilon>0$. By Lemma 4.2 and stationarity, we can select $r$ large enough such that

$$
\gamma_{u, \lambda}=\mathrm{P}_{\lambda}\left(S(u+B)<\lfloor\lambda c\rfloor-r, \sup _{u \leq v \leq u+1} S(v+B) \geq\lfloor\lambda c\rfloor\right) \leq \varepsilon \lambda^{-1 / 2} \mathrm{e}^{-\lambda I}
$$

for all large $\lambda$. Let $k=\left\lfloor m^{1 / 2}\right\rfloor, \Gamma_{m}=\left\{t \in \mathbb{Z}^{d}: k \leq t \leq(m-k)\right\}$, and $\Omega_{m}=\left\{t \in \mathbb{Z}^{d}: 0 \leq\right.$ $t \leq m\} \backslash \Gamma_{m}$. Then

$$
\begin{aligned}
& \sum_{u \in \underline{C}_{m \lambda}-1} \mathrm{P}_{\lambda}(\underbrace{\bigcup}_{\left\{w \in \underline{C}_{m \lambda^{-1}}: w \neq u\right\}}\left(E_{u, m, \lambda} \cap E_{w, m, \lambda}\right)) \\
& \left.\quad \leq \sum_{u \in \underline{C}_{\lambda}-1} \gamma_{u, \lambda}+\sum_{\left\{u, w \in \underline{C}_{m \lambda}-1\right.}: w \neq u\right\} \\
& \mathrm{P}_{\lambda}\left(G_{u, m, \lambda} \cap G_{w, m, \lambda}\right)+\sum_{u \in \underline{C}_{m \lambda}-1} \mathrm{P}_{\lambda}\left(H_{u, m, \lambda}\right),
\end{aligned}
$$

where

$$
\begin{aligned}
& G_{u, m, \lambda}=\mathrm{P}_{\lambda}\left(S(v+B) \geq\lfloor\lambda c\rfloor-r \text { for some } v \in u+\lambda^{-1} \Gamma_{m}\right), \\
& H_{u, m, \lambda}=\mathrm{P}_{\lambda}\left(S(v+B) \geq\lfloor\lambda c\rfloor-r \text { for some } v \in u+\lambda^{-1} \Omega_{m}\right) .
\end{aligned}
$$

By (4.12) and (C.1),

$$
\sum_{u \in \underline{C}_{\lambda}-1} \gamma_{u, \lambda} \leq[\varepsilon+o(1)] \lambda^{d-1 / 2} \mathrm{e}^{-\lambda I} \sigma_{d}(D)
$$


Let $L>\sup _{x, y \in D}\|x-y\|_{\infty}$. Then, by (C.3), stationarity, and Lemma 4.3, there exists $m$ large enough such that, for all $u \in \underline{C}_{m \lambda^{-1}}$ and large $\lambda$,

$$
\begin{aligned}
& \sum_{\left\{w \in \underline{C}_{m \lambda-1}: w \neq u\right\}} \mathrm{P}_{\lambda}\left(G_{u, m, \lambda} \cap G_{w, m, \lambda}\right) \\
& \left.\quad \leq m^{d} \sum_{\left\{v \in\left(\lambda^{-1} \mathbb{Z}\right)^{d}: k \lambda-1\right.} \leq\|v\|_{\infty} \leq L\right\} \\
& \quad \leq[\varepsilon+o(1)] m^{d} \lambda^{-1 / 2} \mathrm{e}^{-\lambda I} .
\end{aligned}
$$

Hence, by (4.12),

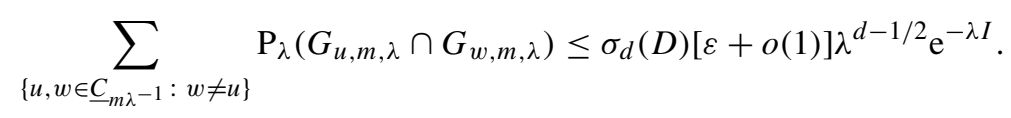

Since $k=\left\lfloor m^{1 / 2}\right\rfloor$, it follows that $\# \Omega_{m}=(m+1)^{d}-(m+1-2 k)^{d}=O\left(m^{d-1 / 2}\right)$. By (4.12), (C.4), and a modification of (4.8) (with $\sum_{\ell=-\infty}^{r}$ in place of $\sum_{\ell=-\infty}^{0}$ ),

$$
\sum_{u \in \underline{C}_{m \lambda}-1} \mathrm{P}_{\lambda}\left(H_{u, m, \lambda}\right)=O\left(m^{d-1 / 2}\left(\lambda m^{-1}\right)^{d} \sigma_{d}(D) \lambda^{-1 / 2} \mathrm{e}^{-\lambda I}\right) \leq \varepsilon \lambda^{d-1 / 2} \mathrm{e}^{-\lambda I} \sigma_{d}(D)
$$

for all large $m$. We then obtain (4.9) from (C.2) and (C.5)-(C.7) by choosing $\varepsilon$ arbitrarily small.

\section{Acknowledgements}

The author thanks the reviewers for their comments which have led to improvements in the presentation of the manuscript. This research was partially supported by NUS grant R-155000-062-112.

\section{References}

[1] Adler, R. J. (2000). On excursion sets, tube formulas and maxima of random fields. Ann. Appl. Prob. 10, 1-74.

[2] Albin, J. M. P. (1990). On extremal theory for stationary processes. Ann. Prob. 18, 92-128.

[3] Alm, S. E. (1997). On the distribution of scan statistics of a two-dimensional Poisson process. Adv. Appl. Prob. 29, 1-18.

[4] Anderson, N. H. And Titterington, D. M. (1997). Some methods for investigating spatial clustering with epidemiological applications. J. R. Statist. Soc. A 160, 87-105.

[5] Berman, S. M. (1982). Sojourns and extremes of stationary processes. Ann. Prob. 10, 1-46.

[6] Bickel, P. And Rosenblatt, M. (1973). Two-dimensional random field. In Multivariate Analysis, III, Academic Press, New York, pp. 3-15.

[7] Chan, H. P. AND LAI, T. L. (2006). Maxima of asymptotically Gaussian random fields and moderate deviation approximations to boundary crossing probabilities of sums of random variables with multidimensional indices. Ann. Prob. 34, 80-121.

[8] Chan, H. P. and Zhang, N. R. (2007). Scan statistics with weighted observations. J. Amer. Statist. Assoc. 102, 595-602.

[9] Cressie, N. (1993). Statistics for Spatial Data. John Wiley, New York.

[10] Feller, W. (1971). An Introduction to Probability Theory and Its Applications, Vol. 2, 2nd edn. John Wiley, New York.

[11] Glaz, J., Naus, J. and Wallenstein, S. (2001). Scan Statistics. Springer, New York.

[12] Hogan, M. and Siegmund, D. O. (1986). Large deviations for the maxima of some random fields. Adv. Appl. Math. 7, 2-22.

[13] Kabluchko, Z. And Spodarev, E. (2009). Scan statistics of Lévy noises and marked empirical processes. Adv. Appl. Prob. 41, 13-37. 
[14] LoAder, C. R. (1991). Large-deviation approximations to the distribution of scan statistics. Adv. Appl. Prob. 23, $751-771$

[15] Naus, J. I. (1965). Clustering of random points in two dimensions. Biometrika 52, 263-267.

[16] PICKands, J., III (1969). Upcrossing probabilities for stationary Gaussian processes. Trans. Amer. Math. Soc. 145, 51-75.

[17] Piterbarg, V. (1996). Asymptotic Methods in the Theory of Gaussian Processes and Fields (Transl. Math. Monogr. 148), American Mathematical Society, Providence, RI.

[18] Qualls, C. and Watanabe, H. (1973). Asymptotic properties of Gaussian random fields. Trans. Amer. Math. Soc. 177, 155-171.

[19] Shafie, K., Sigal, B., Siegmund, D. and Worsley, K. J. (2003). Rotation space random fields with an application to fMRI data. Ann. Statist. 31, 1732-1771.

[20] Siegmund, D. (1985). Sequential Analysis. Springer, New York.

[21] Siegmund, D. O. And Worsley, K. J. (1995). Testing for a signal with unknown location and scale in a stationary Gaussian random field. Ann. Statist. 23, 608-639.

[22] SpIvaK, M. (1965). Calculus on Manifolds: A Modern Approach to Classical Theorems of Advanced Calculus. W. A. Benjamin, New York.

[23] Storey, J. D. And Siegmund, D. O. (2001). Approximate $p$-values for local sequence alignments: numerical studies. J. Comput. Biol. 8, 549-556.

[24] TaYlor, J. E. (2006). A Gaussian kinematic formula. Ann. Prob. 34, 122-158.

[25] Taylor, J., Takemura, A. And Adler, R. J. (2005). Validity of the expected Euler characteristic heuristic. Ann. Prob. 33, 1362-1396.

[26] TU, I.-P. (2009). Asymptotic overshoots for arithmetic i.i.d. random variables. Statistica Sinica 19, $315-323$.

[27] Tu, I. And Siegmund, D. O. (1999). The maxima of a function of a Markov chain and application to linkage analysis. Adv. Appl. Prob. 31, 510-531.

[28] Woodroofe, M. (1979). Repeated likelihood ratio tests. Biometrika 66, 454-463. 